\title{
Image assimilation for motion estimation of atmospheric layers with shallow-water model
}

\author{
Nicolas Papadakis ${ }^{1}$, Patrick Héas ${ }^{1}$ and Étienne Mémin ${ }^{1,2,3}$ \\ ${ }^{1}$ IRISA/INRIA, Campus de Beaulieu 35042 Rennes - FRANCE \\ ${ }^{2}$ CEFIMAS, Avenida Santa Fe 1145 C1059ABF,Buenos Aires - Argentina \\ ${ }^{3}$ Fac. de Ing. de la Univ. Buenos-Aires, Av. Paseo Colón 850, C1063ACV Buenos \\ Aires - Argentina
}

\begin{abstract}
The complexity of dynamical laws governing 3D atmospheric flows associated to incomplete and noisy observations makes very difficult the recovery of atmospheric dynamics from satellite images sequences. In this paper, we face the challenging problem of joint estimation of timeconsistent horizontal motion fields and pressure maps at various atmospheric depths. Based on a vertical decomposition of the atmosphere, we propose a dense motion estimator relying on a multi-layer dynamical model. Noisy and incomplete pressure maps obtained from satellite images are reconstructed according to shallow-water model on each cloud layer using a framework derived from data assimilation. While reconstructing dense pressure maps, this variational process estimates timeconsistent horizontal motion fields related to the multi-layer model. The proposed approach is validated on a synthetic example and applied to a real world meteorological satellite image sequence.
\end{abstract}

\section{Introduction}

Geophysical motion characterization and image sequence analysis are crucial issues for numerous scientific domains involved in the study of climate change, weather forecasting, climate prediction or biosphere analysis. The use of surface station, balloon, and more recently in-flight aircraft measurements and low resolution satellite images has improved the estimation of wind fields and has been a subsequent step for a better understanding of meteorological phenomena. However, the network's temporal and spatial resolutions may be insufficient for the analysis of mesoscale dynamics. Recently, in an effort to avoid these limitations, another generation of satellites sensors has been designed, providing image sequences characterized by finer spatial and temporal resolutions. Nevertheless, the analysis of motion remains particularly challenging due to the complexity of atmospheric dynamics at such scales.

Tools are needed to exploit this new generation of satellite images and we believe that it is very important that the computer vision community gets involved in such domain as they can potentially bring relevant contributions with respect to the analysis of spatio-temporal data.

Nevertheless, in the context of geophysical motion analysis, standard techniques from computer vision, originally designed for bi-dimensional quasi-rigid motions with stable salient features, appear to be not well adapted $[1,2]$. The 
design of techniques dedicated to fluid flow has been a step forward, towards the constitution of reliable methods to extract characteristic features of flows [3, 4]. However, for geophysical applications, existing fluid-dedicated methods are all limited to frame to frame estimation and do not use the underlying physical laws. Moreover, geophysical flows are quite well described by appropriate physical models. As a consequence in such contexts, physic-based approach can be very powerful for analyzing incomplete and noisy image data, in comparison to standard statistical methods. The inclusion of physical a priori leads to unusual advanced techniques for motion analysis which may be of interest for the computer vision community. This yields to new application domains impacting potentially studies of capital interest for our everybody life, and obviously to the devise of proper efficient techniques. This is thus a research domain with wide perspectives. Our work is a contribution towards this direction.

The method proposed in this paper is significantly different from previous works on motion analysis by satellite imagery. Indeed our method estimates physical sound and time consistent motion fields retrieved at different atmospheric levels for the whole image sequence. More precisely, we use a shallow-water formulation of the Navier-Stokes equations to control the motion evolution across the sequence. This is done through a variational approach derived from data assimilation principle which combines the a priori exact dynamic and the pressure difference observations obtained from satellite images.

\section{Data assimilation principle}

\subsection{Introduction}

Data Assimilation is a technique related to optimal control theory which allows estimating over time the state of a system of variables of interest [5-8]. This method enables a smoothing of the unknown variables according to an initial state of the system, a dynamic law and noisy measurements of the system's state.

Let $\mathcal{V}$ be a Hilbert space identified to its dual defined over $\Omega$. The evolution of the state variable $X \in \mathcal{W}\left(t_{0}, t_{f}\right)=\left\{f \mid f \in L^{2}\left(t_{0} ; t_{f} ; \mathcal{V}\right\}\right.$ is assumed to be described through a (possibly non linear) differential dynamical model $\mathbb{M}: \mathcal{V} \mapsto \mathcal{V}$ :

$$
\| \begin{aligned}
& \partial_{t} X(\boldsymbol{x}, t)+\mathbb{M}(X(\boldsymbol{x}, t))=0 \\
& X\left(t_{0}\right)=X_{0}
\end{aligned}
$$

where $X_{0}$ is a control parameter. We then assume that noisy observations $Y \in \mathcal{O}$ are available. These observations may live in a different space (a reduced space for instance) from the state variable. We will nevertheless assume that there exists a differential operator $\mathbb{H}: \mathcal{V} \mapsto \mathcal{O}$, that goes from the variable space to the observation space. A least squares estimation of the control variable regarding the whole sequence of measurements available within a considered time range comes to minimize with respect to the control variable $X_{0} \in \mathcal{V}$, a cost function of the following form:

$$
J\left(X_{0}\right)=\frac{1}{2} \int_{t_{0}}^{t_{f}}\left\|Y-\mathbb{H} X\left(X_{0}\right)\right\|_{R}^{2} d t
$$


where $R$ is the covariance matrix of the observations $Y$. A first approach consists in computing the functional gradient through finite differences. Denoting $N$ the dimension of the control parameter $X_{0}$, such a computation is impractical for control space of large dimension since it requires $N$ integrations of the evolution model for each required value of the gradient functional. Adjoint models as introduced first in meteorology by Le Dimet and Talagrand in [7] authorize the computation of the gradient functional in a single backward integration of an adjoint variable. The value of this adjoint variable at the initial time provides the value of the gradient at the desired point. This first approach is widely used in environmental sciences for the analysis of geophysical flows $[7,8]$.

\subsection{Differentiated model}

To obtain the adjoint model, the system of equations (1) is firstly differentiated with respect to a small perturbation $d X=\frac{\partial X}{\partial X_{0}} d X_{0}$ :

$$
\| \begin{aligned}
& \partial_{t} d X(\boldsymbol{x}, t)+\partial_{X} \mathbb{M} d X=0 \\
& d X\left(t_{0}\right)=d X_{0}
\end{aligned}
$$

where $\partial_{X} \mathbb{M}$ is the tangent linear operator of $\mathbb{M}$ defined by its gâteaux derivative. The gradient of the functional in the direction $d X_{0}$ must also be computed:

$$
\begin{aligned}
\left\langle\frac{\partial J}{\partial X_{0}}, d X_{0}\right\rangle & =\int_{t_{0}}^{t_{f}}\left\langle\left(Y-\mathbb{H} X\left(X_{0}\right), \mathbb{H} \frac{\partial X}{\partial X_{0}} d X_{0}\right\rangle_{R} d t\right. \\
& =\int_{t_{0}}^{t_{f}}\left\langle\mathbb{H}^{*} R\left(Y-\mathbb{H} X\left(X_{0}\right), d X\right\rangle_{\mathcal{V}} d t,\right.
\end{aligned}
$$

where $\mathbb{H}^{*}$ is the adjoint operator of $\mathbb{H}$ defined by:

$$
\forall X \in \mathcal{V}, Y \in \mathcal{O} ;\langle X, \mathbb{H} Y\rangle_{\mathcal{V}}=\left\langle\mathbb{H}^{*} X, Y\right\rangle_{\mathcal{O}} .
$$

\subsection{Adjoint model}

We then introduce the adjoint variable $\lambda \in \mathcal{W}\left(t_{0}, t_{f}\right)$. The first equation of the differentiated model (3) is multiplied by this adjoint variable and integrated in the time interval $\left[t_{0} ; t_{f}\right]$ :

$$
\int_{t_{0}}^{t_{f}}\left\langle\partial_{t} d X(\boldsymbol{x}, t)+\partial_{X} \mathbb{M} d X, \lambda\right\rangle_{\mathcal{V}}=0
$$

After an integration by parts, we have:

$$
\int_{t_{0}}^{t_{f}}\left\langle-\partial_{t} \lambda+\partial_{X} \mathbb{M}^{*} \lambda, d X(\boldsymbol{x}, t)\right\rangle_{\mathcal{V}} d t=\left\langle\lambda\left(t_{0}\right), d X\left(t_{0}\right)\right\rangle_{\mathcal{V}}-\left\langle\lambda\left(t_{f}\right), d X\left(t_{f}\right)\right\rangle_{\mathcal{V}}
$$

where the adjoint operator $\partial_{X} \mathbb{M}^{*}$ is defined by:

$$
\forall X, Y \in \mathcal{V} ;\left\langle X, \partial_{X} \mathbb{M} Y\right\rangle_{\mathcal{V}}=\left\langle\partial_{X} \mathbb{M}^{*} X, Y\right\rangle_{\mathcal{V}} .
$$


To perform the computation of the gradient functional, we assume that $\lambda\left(t_{f}\right)=0$ and define the following adjoint problem:

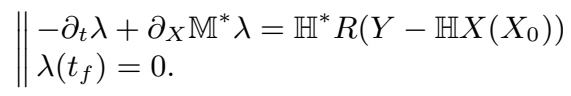

\subsection{Functional gradient}

Combining (4), (5) and (6), we finally obtain the gradient functional as:

$$
\frac{\partial J}{\partial X_{0}}=\lambda\left(t_{0}\right)
$$

Hence, assimilation principle enables to compute the functional gradient with a single backward integration. In the next section, we adapt this process to the control of high dimensional state variables, characterizing the dynamics of layered atmospheric flows.

\section{Application to atmospheric layer motion estimation}

\subsection{Layer decomposition}

The layering of atmospheric flow in the troposphere is valid in the limit of horizontal scales much greater than the vertical scale height, thus roughly for horizontal scales greater than $100 \mathrm{~km}$. In order to make the layering assumption valid in the case of satellite images of kilometer order, low resolution observations relevant of a coarser grid are considered. Thus, one can decompose the 3D space into elements of variable thickness, corresponding to layers. Analysis based on such decomposition presents the main advantage of operating at different atmospheric pressure ranges and avoids the mix of heterogeneous observations.

Let us present the 3D space decomposition that we chose for the definition of the $K$ layers. The $k$-th layer corresponds to the volume lying in between an upper surface $s^{k+1}$ and a lower surface $s^{k}$. These surfaces $s^{k}$ are defined by the height of top of clouds belonging to the $k$-th layer. They are thus defined only in areas where there exists clouds belonging to the $k$-th layer, and remains undefined elsewhere. The membership of top of clouds to the different layers is determined by cloud classification maps. Such classifications which are based on thresholds of top of cloud pressure, are routinely provided by the EUMETSAT consortium, the European agency which supplies the METEOSAT satellite data.

\subsection{Sparse pressure difference observations}

Top of cloud pressure images are also routinely provided by the EUMETSAT consortium. They are derived from a radiative transfer model using ancillary data obtained by analysis or short term forecasts. Multi-channel techniques enable the determination of the pressure at the top of semi-transparent clouds [9]. 
We denote by $C^{k}$ the class corresponding to the $k$-th layer. Note that the top of cloud pressure image denoted by $p_{\cup}$ is composed of segments of top of cloud pressure functions $p\left(s^{k+1}\right)$ related to the different layers. That is to say: $p_{\cup}=\left\{\bigcup_{k} p\left(s^{k+1}, \mathbf{s}\right) ; \mathbf{s} \in C^{k}\right\}$. Thus, pressure images of top of clouds are used to constitute sparse pressure maps of the layer upper boundaries $p\left(s^{k+1}\right)$. As in satellite images, clouds lower boundaries are always occluded, we coarsely approximate the missing pressure observations $p\left(s^{k}\right)$ by an average pressure value $\bar{p}^{k}$ observed on top of clouds of the layer underneath. Finally, for layer $k \in[1, K]$, we define observations $h_{o b s}^{k}$ as pressure differences in Pascal (Pa) units:

$$
h_{o b s}^{k} \begin{cases}=\bar{p}^{k}(\mathbf{s})-p \cup & \text { if } \mathbf{s} \in C^{k} \\ =0 & \text { if } \mathbf{s} \in \bar{C}^{k}\end{cases}
$$

\subsection{Shallow-water model}

In order to provide a dynamical model for the previous pressure difference observations, we use the shallow-water approximation (horizontal motion much greater than vertical motion) derived under the assumption of layer incompressibility (layers are characterized by mean densities $\rho^{k}$ ). The shallow-water approximation is valid for mesoscale analysis in a layered atmosphere. As friction components can be neglected, the vertical integration of the momentum equation between boundaries $s^{k}$ and $s^{k+1}$ yields for the k-th layer to the equation [6, $10,11]$ :

$$
\frac{\partial\left(\mathbf{q}^{k}\right)}{\partial t}+\operatorname{div}\left(\frac{1}{h^{k}} \mathbf{q}^{k} \otimes \mathbf{q}^{k}\right)+\frac{1}{2 \rho^{k}} \nabla_{x y}\left(h^{k}\right)^{2}+g h^{k} \nabla_{x y}\left(s^{k+1}\right)+\left[\begin{array}{cc}
0 & -1 \\
1 & 0
\end{array}\right] f^{\phi} \mathbf{q}^{k}=0
$$

with

$$
\begin{aligned}
h^{k} & =p\left(z=s^{k}\right)-p\left(z=s^{k+1}\right), \\
\mathbf{v}^{k} & =\left(u^{k}, v^{k}\right)=\frac{1}{h^{k}} \int_{p\left(z=s^{k+1}\right)}^{p\left(z=s^{k}\right)} \mathbf{v} d p, \\
\mathbf{q}^{k} & =h^{k} \mathbf{v}^{k}, \\
\operatorname{div}\left(\frac{1}{h^{k}} \mathbf{q}^{k} \otimes \mathbf{q}^{k}\right) & =\left[\frac{\frac{\partial\left(h^{k}\left(u^{k}\right)^{2}\right)}{\partial x}+\frac{\partial\left(h^{k} u^{k} v^{k}\right)}{\partial y}}{\frac{\partial\left(h^{k} u^{k} v^{k}\right)}{\partial x}+\frac{\partial\left(h^{k}\left(v^{k}\right)^{2}\right)}{\partial y}}\right] .
\end{aligned}
$$

By adding the integrated continuity equation to Eq. 9, we obtain independent shallow-water equation systems [11] for layers $k \in[1, K]$ :

$$
\left\{\begin{array}{l}
\left.\frac{\partial h^{k}}{\partial t}+\operatorname{div}\left(\mathbf{q}^{k}\right)\right) \\
\frac{\partial\left(\mathbf{q}^{k}\right)}{\partial t}+\operatorname{div}\left(\frac{1}{h^{k}} \mathbf{q}^{k} \otimes \mathbf{q}^{k}\right)+\frac{1}{2 \rho^{k}} \nabla_{x y}\left(h^{k}\right)^{2}+\left[\begin{array}{cc}
0 & -1 \\
1 & 0
\end{array}\right] f^{\phi} \mathbf{q}^{k}=0,
\end{array}\right.
$$

where we have assumed that surfaces $s^{k}$ and $s^{k+1}$ are locally flat in the vicinity of a pixel. This expression is discretized spatially with non oscillatory schemes [12] and integrated in time with a third order Runge-Kutta scheme. This equation system describes the dynamics of physical quantities expressed in standard units. 
Thus, some dimension factors appear in the equation when it is discretized on a pixel grid with velocities expressed in pixels per frame and pressure in hecto pascal $h P a$. As one pixel represents $\Delta x$ meters and one frame corresponds to $\Delta t$ seconds, the densities $\rho^{k}$ expressed in pascal by square seconds per square meter $\left(\mathrm{Pa} \mathrm{s} \mathrm{s}^{2} / \mathrm{m}^{2}\right)$ must be multiplied by $10^{-2} \Delta x^{2} / \Delta t^{2}$, and coriolis factor $f^{\phi}$ expressed per seconds must be multiplied by $\Delta t$. By a scale analysis and as also observed in our experiments, for $\Delta t=900$ seconds, the third term of equation 9 has a magnitude similar to other terms if $\Delta x \geq 25 \mathrm{~km}$. This is in agreement with the shallow water assumption.

\subsection{Assimilation of layer motion and pressure differences}

We can now define all the components of the assimilation system allowing the recovery of pressure difference observations obtained from section 3.2 through the dynamical model presented in section 3.3. The final system enables the tracking of pressure difference $h^{k}$ and average velocities $\mathbf{q}^{k}$ related to the set of $k \in[1, K]$ layers. Referring to section 2, we then have $X^{k}=\left[h^{k}, \mathbf{q}^{k}\right]^{T}$. The evolution model $\mathbb{M}$ is given by the mesoscale dynamics (14). The only observations available are the pressure difference maps $h_{o b s}^{k}$. For each layer $k$, the observation operator then reads: $\mathbb{H}=[1,0]$ and the process minimizes:

$$
J_{k}\left(h_{0}^{k}, \mathbf{q}_{0}^{k}\right)=\int_{t_{0}}^{t_{f}}\left\|h_{o b s}^{k}-h^{k}\left(h_{0}^{k}, \mathbf{q}_{0}^{k}\right)\right\|_{R^{k}}^{2} d t,
$$

through a backward integrations of the adjoint model $\left(\partial_{X} \mathbb{M}\right)^{*}$ defined by:

$$
\left\{\begin{aligned}
&-\partial_{t} \lambda_{h}^{k}(t)+\boldsymbol{w}^{k} \cdot\left(\boldsymbol{w}^{k} \cdot \nabla\right) \lambda_{\mathbf{q}}^{k}-\frac{h^{k}}{\varrho^{k}} \operatorname{div}\left(\lambda_{\mathbf{q}}^{k}\right)=R^{k}\left(h_{o b s}^{k}(t)-h^{k}(t)\right), \\
&-\partial_{t} \lambda_{\mathbf{q}}^{k}(t)-\left(\boldsymbol{w}^{k} \cdot \nabla\right) \lambda_{\mathbf{q}}^{k}-\left(\nabla \lambda_{\mathbf{q}}^{k}\right) \boldsymbol{w}^{k}-\nabla \lambda_{h}^{k}+\left[\begin{array}{rr}
0 & 1 \\
-1 & 0
\end{array}\right] f^{\phi} \lambda_{\mathbf{q}}^{k}=0 \\
& \lambda_{h}^{k}\left(t_{f}\right)=0 \\
& \lambda_{\mathbf{q}}^{k}\left(t_{f}\right)=0 .
\end{aligned}\right.
$$

In this expression, $\lambda_{h}^{k}$ and $\lambda_{\mathbf{q}}^{k}$ are the two components of the adjoint variable $\lambda^{k}$ of layer $k$ [10]. More details on the construction of adjoint models can be found in $[8]$.

One can finally define a diagonal covariance matrix $R^{k}$ using the mask of observation $C^{k}$ :

$$
R^{k}(\mathbf{s}, \mathbf{s})= \begin{cases}=\alpha & \text { if } \mathbf{s} \in C^{k} \\ =0 & \text { if } \mathbf{s} \in \bar{C}^{k}\end{cases}
$$

where $\alpha$ is a fixed parameter (set to 0.1 in our applications) defining the observation covariances. However, as observations are sparse, a nine-diagonal covariance matrix is employed to diffuse information in a $3 \times 3$ pixel vicinity. As the assimilation process is not insured to reach a global minima, results depend on initialization. Thus, state variables $h_{0}^{k}$ are initialized with a constant value while initial values for variables $\mathbf{q}_{0}^{k}$ are provided by an optic-flow algorithm dedicated to atmospheric layers [3]. 


\section{Results}

\subsection{Synthetic experiments}

For an exhaustive evaluation, we have relied on image observations generated by short time numerical simulation of atmospheric layer motion according to shallow-water dynamical model (Eq. 14). Realistic initial conditions on layer pressure function and motion have been chosen to derive a synthetic sequence of 10 images. The sequence has then been deteriorated by different noises and by a masking operation to form 4 different data sets. The two first synthetic image sequences named $e_{1}$ and $e_{2}$ are thus composed of dense observations of $h_{o b s}^{k}$ in hecto-pascal units $(\mathrm{hPa})$ corrupted by Gaussian noises with standard deviation respectively equal to 10 and $20 \%$ of the pressure amplitude. A real cloud classification map (used in the next experiment) has been employed to extract regions of data sets $e_{1}$ and $e_{2}$ in order to create two noisy and incomplete synthetic sequences $e_{3}$ and $e_{4}$ (see figure 2). For initializing the assimilation system, we have not relied on an optic-flow algorithm in this synthetic case. We have used instead known values of variables $h_{0}^{k}$ and $\mathbf{q}_{0}^{k}$ deteriorated by Gaussian noises. Results of the joint motion-pressure estimation performed by image assimilation are evaluated in table 1.

\begin{tabular}{c|c|c|c|c|c|c|} 
& $\begin{array}{c}\text { Mask } \\
\text { Noise } \\
e_{1}\end{array}$ & $\begin{array}{c}h_{\text {obs }}^{k} \text { RMSE } \\
(\mathrm{hPa})\end{array}$ & $\begin{array}{c}\text { final } \\
h^{k} \text { RMSE } \\
(\mathrm{hPa})\end{array}$ & $\begin{array}{c}\text { initial }\left|\mathbf{v}_{0}^{k}\right| \text { RMSE } \\
(\text { pixel/frame) }\end{array}$ & $\begin{array}{c}\text { final }\left|\mathbf{v}_{0}^{k}\right| \text { RMSE } \\
(\mathrm{pixel} / \text { frame })\end{array}$ \\
\hline$e_{2}$ & & 20 & 22.313880 & 5.904791 & 0.22863 & 0.03457 \\
\hline$e_{3}$ & $\mathrm{x}$ & 10 & 15.627055 & 8.133384 & 0.21954 & 0.05078 \\
\hline$e_{4}$ & $\mathrm{x}$ & 20 & 22.798671 & 10.930078 & 0.21574 & 0.04978 \\
\hline
\end{tabular}

Fig. 1. Numerical evaluation. Decrease of the Root Mean Square Error (RMSE) of estimates $h^{k}$ and $\left|\mathbf{v}_{0}^{k}\right|$ by image assimilation for noisy (experiments $e_{1}, e_{2}, e_{3}$ and $e_{4}$ ) and sparse observations (experiments $e_{3}$ and $e_{4}$ ).

It clearly appears that for noisy observations, the assimilation process induces a significant decrease of the RMSE between real and estimated velocities and pressure. Moreover, this table evaluates and demonstrates the efficiency of the proposed estimator for incomplete and noisy observations for both estimating dense motion fields and reconstructing pressure maps $h^{k}$. Examples of reconstruction for experiments $e_{2}$ and $e_{3}$ are presented in figure 2 .

\subsection{Real meteorological image sequence}

We then turned to qualitative comparisons on a real meteorological image sequence. The benchmark data was composed by a sequence of 10 METEOSAT Second Generation (MSG) images, showing top of cloud pressures with a corresponding cloud classification sequence. The $1024 \times 1024$ pixel images cover an area over the north Atlantic Ocean during part of one day (5-June-2004), at a 

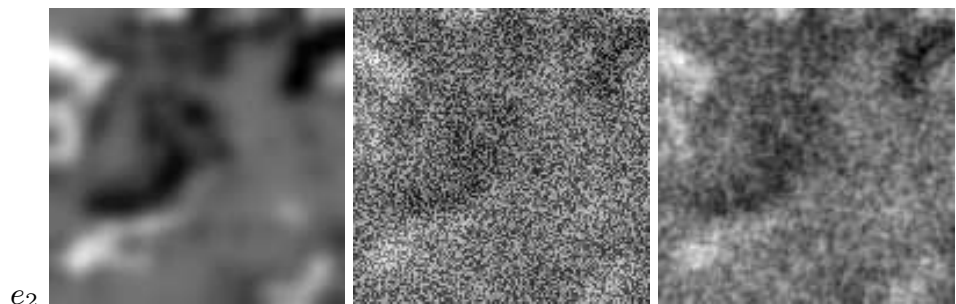

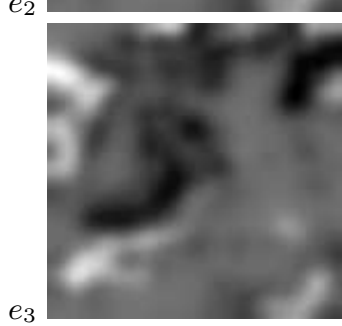

(a) Actual maps

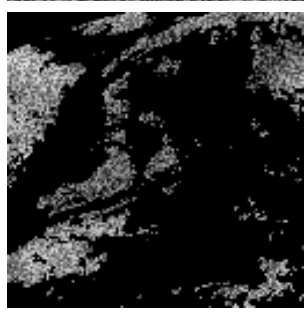

(b) Noised

(and masked) maps

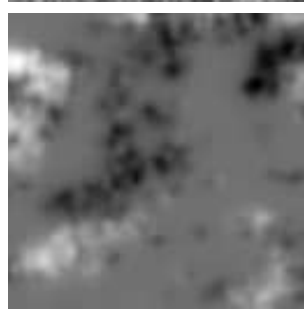

(c) Estimated maps

Fig. 2. Synthetic sequences: Results of experimentations $e_{2}$ and $e_{3}$, where the pressure maps have been noised $\left(e_{2}\right.$ and $\left.e_{3}\right)$ and masked $\left(e_{3}\right)$.

rate of one image every 15 minutes. The spatial resolution is 3 kilometers at the center of the whole Earth image disk. Clouds from a cloud-classification were used to segment images into $K=3$ broad layers, at low, intermediate and high altitude. In order to make the layering assumption valid, low resolution observations on an image grid of $128 \times 128$ pixels are obtained by smoothing and sub-sampling for each layer the original data.

By applying the methodology described in section 3.4 to the image at this coarser resolution, average motion and pressure difference maps are estimated from the image sequence for these 3 layers. Estimated vector fields superimposed on observed pressure difference maps are displayed in figure 3 for each of the 3 layers. The motion fields estimated for the different layers on the cloudy observable parts are consistent with the visual inspection of the sequence. In particular, several motion differences between layers are very relevant. For instance, near the bottom left corner of the images, the lower layer possesses a southward motion while the intermediate layer moves northward. Moreover, the temporal coherence of the retrieved motion demonstrates the efficiency of this spatio-temporal method under physical constraints.

\section{Conclusion}

In this paper, we have presented a new method for estimating time-consistent horizontal winds in a stratified atmosphere from satellite image sequences of top of cloud pressure.

The proposed estimator applies on a set of sparse image observations related to a multi-layer atmosphere, which verify independent shallow-water models. In 
order to manage the incomplete and noisy observations while considering this non-linear physical model, a variational assimilation scheme is proposed. This process estimates time-consistent motion fields related to the layer components while performing the reconstruction of dense pressure difference maps.

The merit of the joint motion-pressure estimator by image assimilation is demonstrated on both synthetic images and real satellite images. In view of the various meteorological studies relying on the analysis of experimental data of atmospheric dynamics, we believe that the proposed multi-layer horizontal wind field estimation technique constitutes a valuable tool.

\section{Acknowledgments}

This work was supported by the European Community through the IST FET Open FLUID Project (http://fluid.irisa.fr)

\section{References}

1. Horn, B., Schunck, B.: Determining optical flow. Artificial Intelligence 17 (1981) $185-203$

2. Leese, J., Novack, C., Clark, B.: An automated technique for obtained cloud motion from geosynchronous satellite data using cross correlation. Journal of applied meteorology 10 (1971) 118-132

3. Héas, P., Mémin, E., Papadakis, N., Szantai, A.: Layered estimation of atmospheric mesoscale dynamics from satellite imagery. IEEE Trans. Geoscience and Remote Sensing (2007)

4. Zhou, L., Kambhamettu, C., Goldgof, D.: Fluid structure and motion analysis from multi-spectrum 2D cloud images sequences. In: Proc. Conf. Comp. Vision Pattern Rec. Volume 2., Hilton Head Island, USA (2000) 744-751

5. Bennet, A.: Inverse Methods in Physical Oceanography. Cambridge University Press (1992)

6. Courtier, P., Talagrand, O.: Variational assimilation of meteorological observations with the direct and adjoint shallow-water equations. Tellus 42 (1990) 531-549

7. Le Dimet, F.X., Talagrand, O.: Variational algorithms for analysis and assimilation of meteorological observations: theoretical aspects. Tellus (1986) 97-110

8. Talagrand, O., Courtier, P.: Variational assimilation of meteorological observations with the adjoint vorticity equation. I: Theory. J. of Roy. Meteo. soc. 113 (1987) $1311-1328$

9. Schmetz, J., Holmlund, K., Hoffman, J., Strauss, B., Mason, B., Gaertner, V., Koch, A., Berg, L.V.D.: Operational cloud-motion winds from meteosat infrared images. Journal of Applied Meteorology 32(7) (1993) 1206-1225

10. Honnorat, M., Le Dimet, F.X., Monnier, J.: On a river hydraulics model and Lagrangian data assimilation. In: International Conference on Adaptive Modeling and Simulation, ADMOS'05, Barcelona (2005)

11. de Saint-Venant, A.: Théorie du mouvement non-permanent des eaux, avec application aux crues des rivières et l'introduction des marées dans leur lit. C. R. Acad. Sc. Paris 73 (1871) 147-154

12. Xu, Z., Shu, C.W.: Anti-diffusive finite difference weno methods for shallow water with transport of pollutant. Journal of Computational Mathematics 24 (2006) $239-251$ 

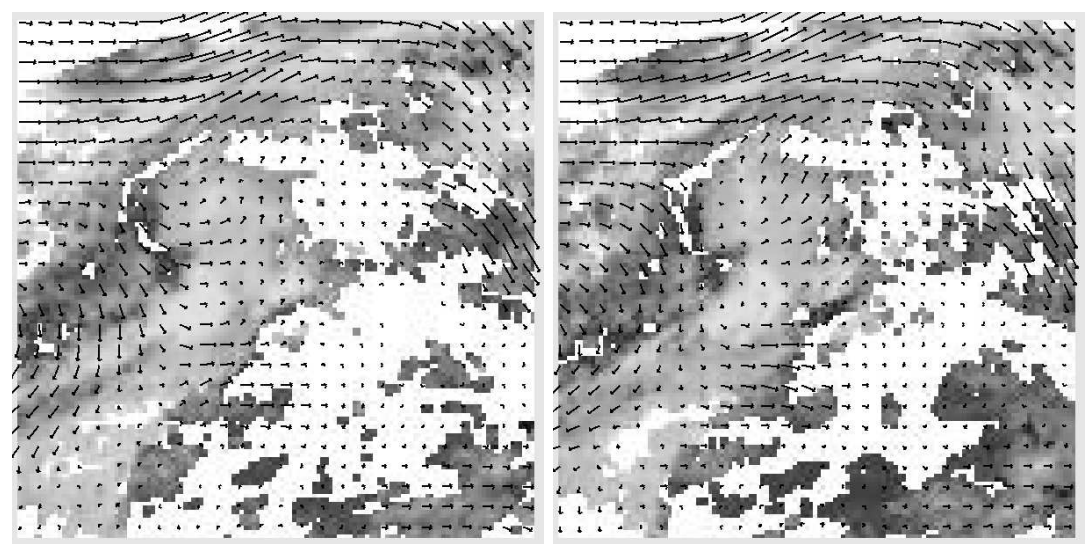

Low layer

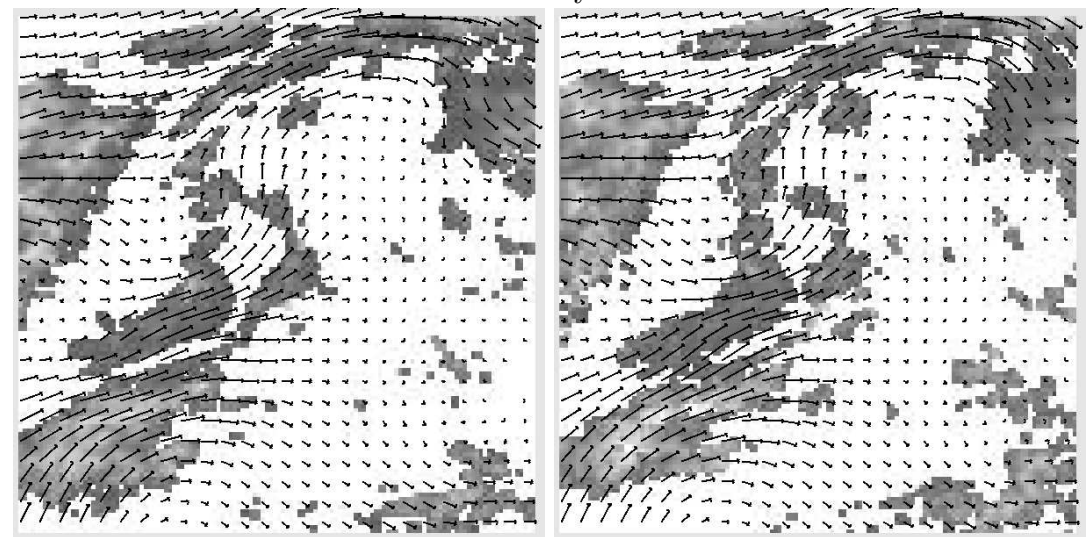

Middle layer

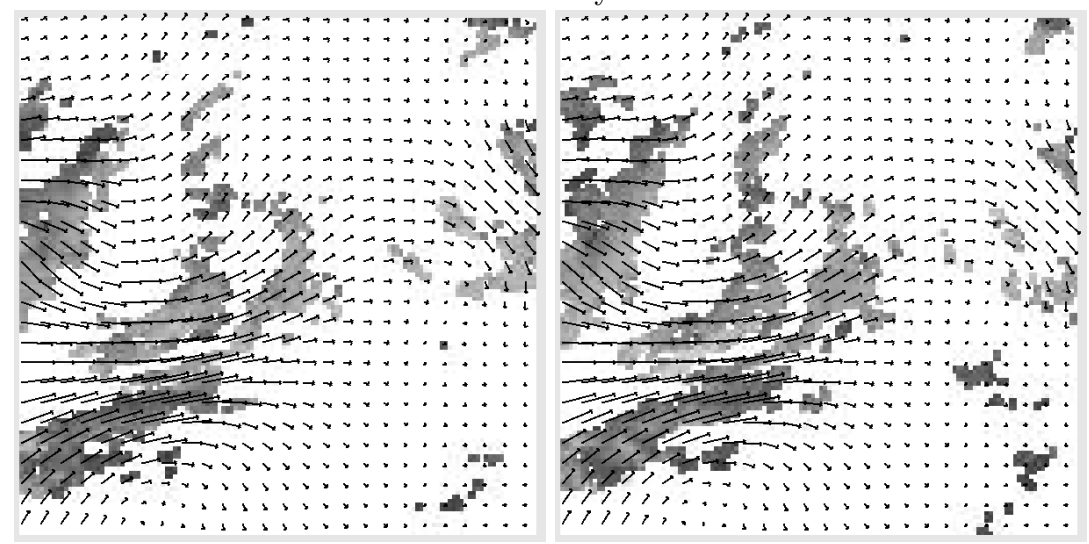

High layer

Fig. 3. First (left) and last (right) estimated horizontal wind fields superimposed on observed pressure difference maps (original images have been subsampled into images of $128 \times 128$ pixels). 